\title{
Design of Software for Maintenance Workforce Scheduling (A Case Study of Afam Power Station, Nigeria)
}

\author{
Ikuobase Emovon ${ }^{1}$, Macaulay Thomas Lilly'ㄹ, Stephen Ogajiye Tamuno-Ojuemi Ogaji ${ }^{3}$ \\ ${ }^{1}$ Mechanical Engineering Department, Federal University of Petroleum Resources, Effurun, Nigeria \\ ${ }^{2}$ Mechanical Engineering Department, Rives State University of Science and Technology, Port Harcourt, Nigeria \\ ${ }^{3}$ School of Engineering, Cranfied University, Bedfordshire, United Kingdom \\ Email: ikuoy2k@yahoo.com
}

Received September 10, 2011; revised December 1, 2011; accepted December 8, 2011

\begin{abstract}
This paper is concerned with the optimization of the workforce scheduling for solving maintenance problems. To achieve this aim an optimsation software for $(5,7)$ problem was developed. The programme was written in Quick Basic. The software was designed to produce a seven day schedule for organization operating a seven day week. Hence organization operating a five day schedule wishing to change to a seven day schedule we find this software very useful. The Quick-Basic computer programme was based on Alfares [1,2] algorithm for solving $(5,7)$ schedule problem. Data collected from Afam power station, Nigeria was used as input data. The test result shows the software is capable of determining workforce size and assigning workers to day-off pattern. The seven-day schedule produced savings of $11 \%$ maintenance labour cost when compared with the 5-day schedule currently being practiced by the Power station [3].
\end{abstract}

Keywords: Optimization; Workforce Scheduling; Software; Quickbasic

\section{Introduction}

For the past ten years, the federal government of Nigeria has embarked on aggressive rural electrification projects across the country. This laudable project is aimed at providing electricity to the rural dwellers where about $64 \%$ of the entire population lives. With this laudable project the federal government hope to minimize, the rural-urban migration thereby forcing young school leavers and graduates to remain in the village self-employed rather than moving to the urban area seeking white-collar jobs [4].

However the pace of this project has drastically slowed down of recent for obvious reasons; 1) Economic meltdown; 2) Drastic decline in the power generation in Nigeria, that is from 5924.7 MW which is the total installed capacity of our generating unit to about $2400 \mathrm{MW}$.

The available mega watt of electricity generated cannot even carter for the urban centers talk less of extending it to rural areas. The negative implication of this power problem in Nigeria has gone beyond rural-urban migration. Most youth that would have been self employed in Nigeria now go into prostitution and drug trafficking, in Asian, Europe and America.

However the major reason why power stations in $\mathrm{Ni}$ geria are generating far below installed capacity is due to maintenance problem. This has affected the availability and reliability of the power plants. In order to reduce these maintenance problems, there is need for effective maintenance scheduling. Scheduling is a crucial component of maintenance management. Maintenance requires three resources: Manpower, materials and equipment. Labour is usually the most important and expensive resource of any organization. Effective labour scheduling is a major determinant of the productivity of the workforce. Improved labour productivity results in efficient handling of maintenance activities. This will invariably result to improved availability and reliability of power plant. This will boost power generation in Nigeria.

Labour scheduling problems are classified into three types 1) shift, or time-of-day, scheduling, 2) days-off, or day-of-week, scheduling, and 3) tour scheduling, which combines the first two types [5]. For organization operating 7 days a week, such as Afam power station, Nigeria, employee days-off scheduling is a significant and relevant problem. The most usual type of days-off schedules is referred to as the $(5,7)$ problem in which each work pattern includes 5 workdays and 2 consecutive off-days per week.

This paper is concerned with development of an Optimisation software for the $(5,7)$ problem using Alfares $[1,2]$ algorithm. The software is capable of determining workforce size and assigning workers to days-off patterns. This software is capable of solving the maintenance manpower scheduleing problem in Afam power station and other Power Station in Nigeria and invariably 
improving Power generation. However, it is not only applicable to power station in Nigeria, but applicable to other organization operating 7 day a week like restaurants, Hospitals and police stations.

The beauty of this software is that it does not require specialize training unlike integer programming software.

This paper is organized as follows: First a review of problem and background of Afam power station. Then Alfares algorithm for solving $(5,7)$ problem is presented. This is followed by computer programme written in quick basic for solving $(5,7)$ problem based on Alfares algorithm. Subsequently result of computer programme as applied to Afam power station maintenance labour scheduling problem. Finally conclusions.

\section{Problem and Background}

From the data collected by Emovon, [6] from Afam Power Station Nigeria it's on record that the first major gas turbine station built in Nigeria is the Afam Gas Turbine Power Station. The power station is located in the Niger Delta because of the large reserve of natural gas in the region. The first phase of the power station was commissioned in 1963 as Afam I, consisting of four generating units with total capacity of $54 \mathrm{MW}$. Consumption growth experienced in the seventies necessitated the construction of Afam II, which was commissioned in 1976. Afam II consists of four additional generating units with installed capacity totaling $92 \mathrm{MW}$. This was followed by Afam III, which was commissioned in 1978, consisting of four units with installed capacity totalling 108 MW. In 1982, six generating units were commissioned as Afam IV with total capacity of $450 \mathrm{MW}$. Finally, Afam V was commissioned in 2002, made up of two units with a total capacity of $138 \mathrm{MW}$. This brought the number of units in Afam power station to 20, with a total installed capacity of $980 \mathrm{MW}$. With only $413 \mathrm{MW}$ currently being produced, the power generated is less than $43 \%$ of the installed capacity.

The station has been generating power far below installed capacity due to maintenance problems. These problems have affected the availability and reliability of the power plant. In order to minimize these maintenance problems, there is a need for effective maintenance scheduling, which is a major determinant of the productivity of the workforce. Improved labour productivity leads to more efficient handling of maintenance activities. Hence the purpose of this paper is the optimization of the workforce scheduling for solving maintenance problems. To achieve this aim, an optimization software for $(5,7)$ problem was developed. The program is written in QuickBasic.

Afam power station maintenance line comprises of the following departments: Mechanical, Electrical, Instrumentation \& Control, Workshop Services, and Planning.
The maintenance management functions in Afam Power Station include both reactive (run-to-failure) and preventive maintenance (PM). The PM type in place is time based, which is not effectively carried out, not to talk of practicing the state-of-the-art, predictive maintenance.

The preventive maintenance procedure and intervals are not well defined. Poor plant history records make it difficult to retrieve PM history and reports of plant/ equipment, especially for old plants. The power plant maintenance in Afam power station is performed by combined effort of the various departments that make up the maintenance line. The maintenance crews of the various departments carry out daily plant maintenance checks. The checks involve taking plant parameters like load, compressor inlet temperature, and plant evaluated operating hours to mention but a few. Apart from daily plant checks, maintenance line crews carry out 3 types of inspections:

1) First minor inspection, also referred to as small or type A inspection;

2) Second minor inspection, also referred to as normal or type B inspection;

3) Major inspection, or type $C$ inspection.

The first minor inspection of the plant is carried out every 4000 evaluated operating hours (EOH). The second minor inspection is carried out every $8000 \mathrm{EOH}$.

The major inspection is usually carried out every $16,000 \mathrm{EOH}$.

Eight mechanical workers are needed for type A inspection on a particular plant, 8 - 10 workers are needed for type B inspection, while 15 workers are needed for type $\mathrm{C}$ inspection. For daily plant checks, only one maintenance worker is needed to handle a plant. Information from plant daily checks, inspections, and other parameters are used by the planning department to develop an annual maintenance schedule.

This study is concerned with scheduling the Mechanical Department (MD) maintenance line, which is in charge of inspections and daily plant maintenance, checks of the mechanical components of the power plant. Based on inspection requirements and past experience, the maintenance engineer of the MD estimated the number of workers needed to satisfy the daily routine of maintenance activities for a typical week. As shown in Table 1, the plant maintenance needs up to 16 workers daily for the morning shift (8:00 am - 4:00 pm), and up to 7 workers daily for each of the afternoon shift (4:00 pm - 12:00 $\mathrm{am})$ and the night shift (12:00 am - 8:00 am).

\section{Models for Scheduling Days-Off}

Alfares [1,2] developed algorithms to minimize the number of workers for $(5,7)$ problem. Each worker must work for 5 days per week and only consecutive pairs of off days are allowed. This algorithms is applicable to 
Table 1. Daily maintenance labour demands for the three shifts.

\begin{tabular}{ccccccccc}
\hline Day i & Mo-1 & Tu-2 & We - 3 & Th - 4 & Fr - 5 & Sa - 6 & Su - 7 \\
\hline $\begin{array}{c}\text { Morning } \\
\text { labour } \\
\text { demand }\end{array}$ & 16 & 16 & 16 & 16 & 16 & 8 & 7 \\
$\begin{array}{c}\text { Afternoon } \\
\text { labour } \\
\text { demand }\end{array}$ & 6 & 6 & 7 & 7 & 7 & 6 & 6 \\
$\begin{array}{c}\text { Night } \\
\text { labour }\end{array}$ & 6 & 6 & 7 & 7 & 7 & 6 & 6 \\
demand & & & & & & & & \\
\hline
\end{tabular}

Afam power station with respect to providing an alternative workforce schedule as well as other organization operating a seven day a week. The integer linear programming (ILP) formulation is given as follows:

$$
\text { Minimize } w=\sum_{i=1}^{7} c_{j} x_{j}
$$

Subject to

$$
\begin{aligned}
& \left(\sum_{j=1}^{7} x_{j}\right)-x_{i}-x_{i-1} \geq r_{i}, \quad i=1, \cdots, 7 \\
& x_{j} \geq 0 \text { and an integer, } j=1, \cdots, 7
\end{aligned}
$$

where:

$\mathrm{x}_{\mathrm{j}}=$ number of workers, assigned to a days off pattern $\mathrm{j}$ i.e. number of workers off on days, $\mathrm{j}$ and $\mathrm{j}+1$.

$\mathrm{c}_{\mathrm{j}}=$ weekly cost of days-off pattern $\mathrm{j}$ per employee, with premium pay for weekend workdays.

$\mathrm{r}_{\mathrm{i}}=$ number of workers required on day $\mathrm{i}$.

$\mathrm{w}=$ workforce size, that is total number of workers assigned to days-off patterns.

Alfares [1,2] further stated that since $\sum_{j=1}^{7} x_{j}$ is equal to w, Equation (2) can be written as:

$$
x_{i}+x_{i-1} \leq b_{i}, i=1, \cdots, 7
$$

whereas

$$
\mathrm{b}_{\mathrm{i}}=\mathrm{w}-\mathrm{r}_{\mathrm{i}}
$$

$=$ maximum number of workers off on day $i$.

Alfares [1] developed a simple equation that yields the minimum workforce size $\mathrm{W}$ for the $(5,7)$ problem. The expression he obtained for minimum workforce size $\mathrm{W}$ is:

$$
\mathrm{W}=\sum_{\mathrm{j}=1}^{7} \mathrm{x}_{\mathrm{j}}=\max \left\{\mathrm{r}_{\max },\left\lceil\frac{1}{5} \sum_{i=1}^{7} \mathrm{r}_{\mathrm{i}}\right\rceil,\left\lceil\frac{\mathrm{R}_{\max }}{3}\right\rceil\right\}
$$

where $r_{\max }=\max \left\{r_{1}, r_{2}, \cdots, r_{7}\right\}$

$$
\mathrm{R}_{\text {max }}=\max \left\{\mathrm{R}_{1}, \mathrm{R}_{2}, \cdots, \mathrm{R}_{7}\right\}
$$

$\lceil\mathrm{a}\rceil=$ Smallest integer $\geq \mathrm{a}$

$$
\mathrm{R}_{\mathrm{i}}=\sum_{\mathrm{j} \in \mathrm{s}_{\mathrm{i}}} \mathrm{r}_{\mathrm{j}} \mathrm{i}=1,2, \cdots, 7
$$

$$
\begin{aligned}
& \mathrm{S}_{\mathrm{i}}=\text { Set of } 4 \text { subscripts } \subset\{1,2,3,4,5,6,7\}, \\
& \mathrm{i}=1,2, \cdots, 7
\end{aligned}
$$

set $S_{1}$ to $S_{7}$ are shown in Table 2 .

After determining the workforce size, he uses a simple algorithm to determine the number of workers assigned to each days-off pattern, $x_{1}, \cdots, x_{7}$. The result are shown in Table 3. Hence completing the optimum solution of the $(5,7)$ problem without linear or interger programming software.

\section{Algorithm Step [2]}

1) Determine the minimum workforce size $W$ using Equation (6) $\mathrm{W}=\max \left\{\mathrm{r}_{\max },\left\lceil\sum \mathrm{r}_{\mathrm{i}} / 5\right\rceil,\left\lceil\mathrm{R}_{\max } / 3\right\rceil\right\}$. In the case of ties go to step 3 . then:

2) (a) If $\max \left\{\mathrm{r}_{\max },\left\lceil\sum \mathrm{r}_{\mathrm{i}} / 5\right\rceil,\left\lceil\mathrm{R}_{\max } / 3\right\rceil\right\}=\left\lceil\mathrm{R}_{\max } / 3\right\rceil$,

- if $\mathrm{R}_{\max } / 3$ is not integer, increment $\mathrm{R}_{\mathrm{i}}=\mathrm{R}_{\max }$ by (3W $-\mathrm{R}_{\max }$ ) to make it a multiple of 3; among the four daily labor demands $r_{j}$, j E $S_{i}$, that can be increased, avoid whenever possible: 1) weekend, i.e., $\mathrm{r}_{6}$ and $\mathrm{r}_{7}$; and 2 ) the maximum labor demand $r_{\max }$.

- Calculate $b_{1}, b_{2}, \cdots, b_{7}$ using Equation (5), then apply system No. i, $i=1, \cdots, 7$, in Table 3 to find $x_{1}, x_{2}, \cdots$, $\mathrm{x}_{7}$. If there are ties for maximum value $\mathrm{R}_{\mathrm{i}}=\mathrm{R}_{\max }$, use any of the systems No. i corresponding to the applicable values of the index i arbitrarily.

then:

(b) If $\max \left\{\mathrm{r}_{\max },\left\lceil\mathrm{r}_{\mathrm{i}} / 5\right\rceil,\left\lceil\mathrm{R}_{\max } / 3\right\rceil\right\}=\left\lceil\sum \mathrm{r}_{\mathrm{i}} / 5\right\rceil$,

- if $\sum \mathrm{r}_{\mathrm{i}} / 5$ is not integer, increment $\sum \mathrm{r}_{\mathrm{i}}$ by $\left(5 \mathrm{~W}-\sum \mathrm{r}_{\mathrm{i}}\right)$ in order to make it a multiple of 5 ; among all seven daily labor demands $r_{1}, \cdots, r_{7}$, chose the ones to be increased according to the criteria given above in step 2(a).

- calculate $b_{1}, b_{2}, \cdots, b_{7}$ using Equation (5), then apply system No. 8 in Table 3 to find $\mathrm{x}_{1}, \mathrm{x}_{2}, \cdots, \mathrm{x}_{7}$.

(c) If $\max \left\{\mathrm{r}_{\text {max }},\left\lceil\sum \mathrm{r}_{\mathrm{i}} / 5\right\rceil,\left\lceil\mathrm{R}_{\text {max }} / 3\right\rceil\right\}=r_{\text {max }}$, then:

- calculate $b_{1}, b_{2}, \cdots, b_{7}$ using Equation (5), then apply system No. 9 in Table 3 to find $x_{1}, x_{2}, \cdots, x_{7}$.

Table 2. Sets of subscripts define by Equation (8) and their complement [1].

\begin{tabular}{lc}
\hline $\mathrm{i}$ & $\mathrm{S}_{\mathrm{i}}$ \\
\hline 1 & $1,2,4,6$ \\
2 & $2,3,5,7$ \\
3 & $3,4,6,1$ \\
4 & $4,5,7,2$ \\
5 & $5,6,1,3$ \\
6 & $6,7,2,4$ \\
7 & $7,1,3,5$ \\
\hline
\end{tabular}


Table 3. Values of Days off Assignment, $x_{1}, \cdots, x_{7}$ for all possible values of W. [2].

\begin{tabular}{|c|c|c|c|c|c|c|c|c|}
\hline No & $\mathrm{w}$ & $\mathrm{x}_{1}$ & $\mathrm{x}_{2}$ & $\mathrm{x}_{3}$ & $\mathrm{x}_{4}$ & $\mathrm{x}_{5}$ & $\mathrm{x}_{6}$ & $\mathrm{x}_{7}$ \\
\hline 1 & $\left\lceil\mathrm{R}_{1} / 3\right\rceil$ & 0 & $b_{2}$ & $b_{4}-x_{4}$ & $\begin{array}{c}\min \\
\left\{b_{4}, b_{5}-x_{5}\right\}\end{array}$ & $\mathrm{b}_{6}-\mathrm{x}_{6}$ & $\begin{array}{c}\min \\
\left\{b_{6}, b_{7}-b_{1}\right\}\end{array}$ & $\mathrm{b}_{1}$ \\
\hline 2 & $\left\lceil\mathrm{R}_{2} / 3\right\rceil$ & $\mathrm{b}_{2}$ & 0 & $\mathrm{~b}_{3}$ & $b_{5}-x_{5}$ & $\min _{\left\{b_{5}, b_{6}-x_{6}\right\}}$ & $\min _{\left\{b_{7}, R_{2}-R_{6}\right\}}$ & $\mathrm{b}_{7}-\mathrm{x}_{6}$ \\
\hline 3 & $\left\lceil\mathrm{R}_{3} / 3\right\rceil$ & $b_{1}-x_{7}$ & $\mathrm{~b}_{3}$ & 0 & $\mathrm{~b}_{4}$ & $\mathrm{~b}_{6}-\mathrm{x}_{6}$ & $\min _{\left\{\mathrm{b}_{6}, \mathrm{R}_{3}-\mathrm{R}_{6}\right\}}$ & $\min _{\left\{b_{1}, b_{7}-x_{6}\right\}}$ \\
\hline 4 & $\left\lceil\mathrm{R}_{4} / 3\right\rceil$ & $\min _{\left\{b_{1}, b_{2}\right\}}$ & $\mathrm{b}_{2}-\mathrm{x}_{1}$ & $\mathrm{~b}_{4}$ & 0 & $\mathrm{~b}_{5}$ & $\min _{\left\{b_{7}, b_{6}-b_{5}\right\}}$ & $\mathrm{b}_{7}-\mathrm{x}_{6}$ \\
\hline 5 & $\left\lceil\mathrm{R}_{5} / 3\right\rceil$ & $b_{1}-x_{7}$ & $\min _{\left\{b_{2}, b_{3}\right\}}$ & $b_{3}-x_{2}$ & $\mathrm{~b}_{5}$ & 0 & $\mathrm{~b}_{6}$ & $\min _{\left\{b_{1}, b_{7}-b_{6}\right\}}$ \\
\hline 6 & $\left\lceil\mathrm{R}_{6} / 3\right\rceil$ & $\min _{\left\{b_{1}-b_{7}, b_{2}\right\}}$ & $\min _{\left\{\mathrm{b}_{2}, \mathrm{R}_{6}-\mathrm{R}_{2}\right\}}$ & $\min _{\left\{b_{4}, b_{3}-x_{2}\right\}}$ & $b_{4}-x_{3}$ & $\mathrm{~b}_{6}$ & 0 & $\mathrm{~b}_{7}$ \\
\hline 7 & $\left\lceil\mathrm{R}_{7} / 3\right\rceil$ & $\mathrm{b}_{1}$ & $\mathrm{~b}_{3}-\mathrm{x}_{3}$ & $\min _{\left\{b_{3}, b_{4}-x_{4}\right\}}$ & $b_{5}-x_{5}$ & $\min _{\left\{b_{5}, b_{6}-b_{7}\right\}}$ & $\mathrm{b}_{7}$ & 0 \\
\hline 8 & $\left\lceil\sum \mathrm{r}_{\mathrm{i}} / 3\right\rceil$ & $\mathrm{w}-\mathrm{b}_{3}-\mathrm{b}_{5}-\mathrm{b}_{7}$ & $\mathrm{~b}_{2}-\mathrm{x}_{1}$ & $b_{3}-x_{2}$ & $\mathrm{~b}_{4}-\mathrm{x}_{3}$ & $b_{5}-x_{4}$ & $\mathrm{~b}_{6}-\mathrm{x}_{5}$ & $\mathrm{~b}_{7}-\mathrm{x}_{6}$ \\
\hline 9 & $\mathrm{r}_{\max }$ & $\begin{array}{c}\min _{\left\{\mathrm{b}_{1}-\mathrm{x}_{7}, \mathrm{~b}_{2}-\mathrm{x}_{2},\right.} \\
\left.\mathrm{w}-\sum_{\mathrm{i}=2}^{7} \mathrm{x}_{\mathrm{i}}\right\}\end{array}$ & $\begin{array}{c}\min _{\left\{b_{2}, b_{3}-x_{3}\right.}, \\
\left.w-\sum_{i=3}^{7} x_{i}\right\}\end{array}$ & $\begin{array}{c}\min _{\left\{\mathrm{b}_{3}, \mathrm{~b}_{4}-\mathrm{x}_{4},\right.} \\
\left.\mathrm{w}-\sum_{\mathrm{i}=4}^{7} \mathrm{x}_{\mathrm{i}}\right\}\end{array}$ & $\begin{array}{c}\min _{\left\{b_{4}, b_{5}-x_{5},\right.} \\
\left.w-\sum_{i=5}^{7} x_{i}\right\}\end{array}$ & $\begin{array}{c}\min _{\left\{\mathrm{b}_{5}, \mathrm{~b}_{6}-\mathrm{x}_{6}\right.}, \\
\left.\mathrm{w}-\mathrm{x}_{6}\right\}\end{array}$ & $\min _{\left\{\mathrm{b}_{6}, \mathrm{~b}_{7}\right\}}$ & $\begin{array}{c}\min _{\left\{\mathrm{b}_{1}, \mathrm{~b}_{7}-\mathrm{x}_{6}\right.}, \\
\left.\mathrm{w}-\mathrm{x}_{5}-\mathrm{x}_{6}\right\}\end{array}$ \\
\hline
\end{tabular}

3) In the case of tiers for $r_{\max },\left\lceil\sum r_{i} / 5\right\rceil,\left\lceil R_{\max } / 3\right\rceil$, choose the argument that needs the minimum total increment and go to the corresponding step: 2(a) for $\left\lceil\mathrm{R}_{\max } / 3\right\rceil$, 2(b) for $\left[\sum \mathrm{r}_{\mathrm{i}} / 5\right]$, and 2(c) for $\mathrm{r}_{\max }$. While $r_{\max }$ does not need incrementing, the increment for $\left[\sum \mathrm{r}_{\mathrm{i}} / 5\right\rceil$ is $\left(5 \mathrm{~W}-\sum \mathrm{r}_{\mathrm{i}}\right)$, and the increment for $\left\lceil\mathrm{R}_{\max } / 3\right\rceil$ is $\left(3 \mathrm{~W}-\mathrm{R}_{\max }\right)$.

For easy execution of the above algorithm a simple computer programme is written in QUICK BASIC.

\section{Computer Program for Solving $(5,7)$ Problem Based on Alfares [1,2] Algorithm}

DECLARE SUB DRIM ()

DECLARE SUB PRINTALL ()

DECLARE SUB XRI ()

DECLARE FUNCTION SRIMIN! (A!, B!)

DECLARE SUB XR ()

DECLARE FUNCTION RMIN! (A!, B!, C!)

DECLARE SUB DAILYREQUIREMENT ()

DECLARE SUB CALBS ()

DECLARE SUB WORKFORCE ()

CLS

DIM SHARED RI(7), R1(7), R2(7), R3(7), R4(7), R5(7), R6(7), R7(7), SUMR(7), B(7)

DIM SHARED WF, RIMAX, SUMRi5, SUMRMAX, IT\$, SUMRi, X(7)

DIM SHARED FLAG AS STRING

PRINT "ENTER DAILY LABOUR

REQUIREMENT"

INPUT "ENTER LABOUR REQUIREMENT FOR MON: ", RI(1)

INPUT "ENTER LABOUR REQUIREMENT FOR TUE: ", RI(2)
INPUT "ENTER LABOUR REQUIREMENT FOR WED: ", RI(3)

INPUT "ENTER LABOUR REQUIREMENT FOR THU: ", RI(4)

INPUT "ENTER LABOUR REQUIREMENT FOR FRI: ", RI(5)

INPUT "ENTER LABOUR REQUIREMENT FOR SAT: ", RI(6)

INPUT "ENTER LABOUR REQUIREMENT FOR SUN: ", RI(7)

CALL DAILYREQUIREMENT

RiMAX $=0$

FOR I $=1$ TO 7

IF RI(I) > RiMAX THEN

END IF

$$
\text { RiMAX }=\text { RI(I) }
$$

NEXT I

SUMRi $=0$

FOR I = 1 TO 7

\section{NEXT I}

$$
\text { SUMRi }=\text { RI(I) + SUMRi }
$$

SUMRi5 = SUMRi / 5 !

SUMRMAX $=0$

FOR I = 1 TO 7

IF SUMR(I) > SUMRMAX THEN

SUMRMAX = SUMR(1)

SELECT CASE I

CASE 1

IT\$ = "R1"

CASE 2

IT\$ = "R2"

CASE 3

IT\$ = "R3"

CASE 4 


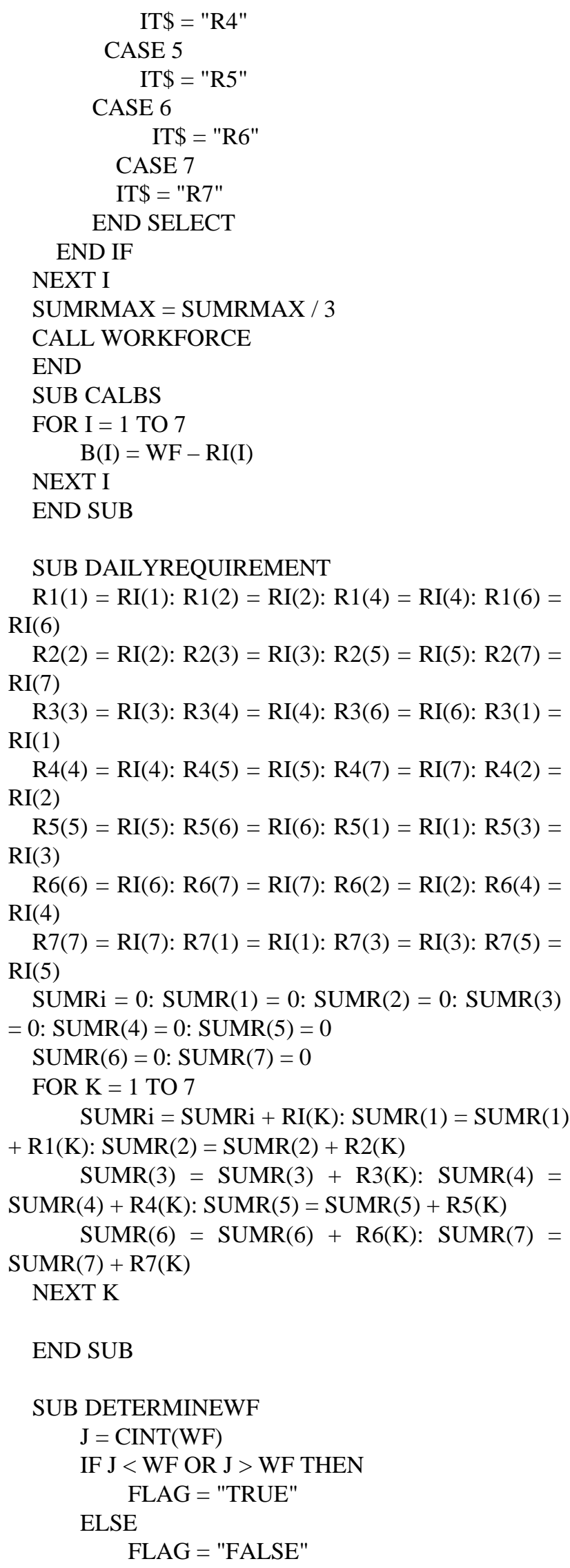

END IF

END SUB

SUB DRIM

IF IT\$ = "R1" THEN

$\mathrm{X}(7)=\mathrm{B}(1)$

$\mathrm{X}(6)=\operatorname{SRIMIN}(\mathrm{B}(6), \mathrm{B}(7)-\mathrm{B}(1))$

$\mathrm{X}(5)=\mathrm{B}(6)-\mathrm{X}(6)$

$\mathrm{X}(4)=\operatorname{SRIMIN}(\mathrm{B}(4), \mathrm{B}(5)-\mathrm{X}(5))$

$\mathrm{X}(3)=\mathrm{B}(4)-\mathrm{X}(4)$

$\mathrm{X}(2)=\mathrm{B}(2)$

$\mathrm{X}(1)=0$

ELSEIF IT\$ = "R2" THEN

$\mathrm{X}(1)=\mathrm{B}(2)$

$\mathrm{X}(2)=0$

$\mathrm{X}(3)=\mathrm{B}(3)$

$\mathrm{X}(6)=\operatorname{SRIMIN}(\mathrm{B}(7), \operatorname{SUMR}(2)-\operatorname{SUMR}(6))$

$\mathrm{X}(5)=\operatorname{SRIMIN}(\mathrm{B}(5), \mathrm{B}(6)-\mathrm{X}(6))$

$\mathrm{X}(4)=\mathrm{B}(5)-\mathrm{X}(5)$

$\mathrm{X}(7)=\mathrm{B}(7)-\mathrm{X}(6)$

ELSEIF IT\$ = "R3" THEN

$\mathrm{X}(2)=\mathrm{B}(3)$

$\mathrm{X}(3)=0$

$\mathrm{X}(4)=\mathrm{B}(4)$

$\mathrm{X}(6)=\operatorname{SRIMIN}(\mathrm{B}(6), \operatorname{SUMR}(3)-\operatorname{SUMR}(6))$

$\mathrm{X}(7)=\operatorname{SRIMIN}(\mathrm{B}(1), \mathrm{B}(7)-\mathrm{X}(6))$

$\mathrm{X}(5)=\mathrm{B}(6)-\mathrm{X}(6)$

$\mathrm{X}(1)=\mathrm{B}(1)-\mathrm{X}(7)$

ELSEIF IT\$ = "R4" THEN

$\mathrm{X}(2)=\mathrm{B}(2)-\mathrm{X}(1)$

$\mathrm{X}(3)=\mathrm{B}(4)$

$\mathrm{X}(4)=0$

$\mathrm{X}(5)=\mathrm{B}(5)$

$\mathrm{X}(6)=\operatorname{SRIMIN}(\mathrm{B}(7), \mathrm{B}(5)-\mathrm{B}(6))$

$\mathrm{X}(7)=\mathrm{B}(7)-\mathrm{X}(6)$

ELSEIF IT\$ = "R5" THEN

$\mathrm{X}(7)=\operatorname{SRIMIN}(\mathrm{B}(1), \mathrm{B}(7)-\mathrm{B}(6))$

$\mathrm{X}(2)=\operatorname{SRIMIN}(\mathrm{B}(2), \mathrm{B}(3))$

$\mathrm{X}(1)=\mathrm{B}(1)-\mathrm{X}(7)$

$\mathrm{X}(3)=\mathrm{B}(3)-\mathrm{X}(2)$

$\mathrm{X}(5)=0$

$\mathrm{X}(4)=\mathrm{B}(5)$

$\mathrm{X}(6)=\mathrm{B}(6)$

ELSEIF IT\$ = "R6" THEN

$\mathrm{X}(2)=\operatorname{SRIMIN}(\mathrm{B}(2), \operatorname{SUMR}(6)-\operatorname{SUMR}(2))$

$\mathrm{X}(3)=\mathrm{SRIMIN}(\mathrm{B}(4), \mathrm{B}(3)-\mathrm{X}(2))$

$\mathrm{X}(4)=\mathrm{B}(4)-\mathrm{X}(3)$

$\mathrm{X}(1)=\mathrm{B}(2)-\mathrm{X}(1)$

$\mathrm{X}(6)=0$

$\mathrm{X}(7)=\mathrm{B}(7)$

ELSE

$\mathrm{X}(5)=\mathrm{B}(6)$

$\mathrm{X}(5)=\operatorname{SRIMIN}(\mathrm{B}(5), \mathrm{B}(6)-\mathrm{B}(7))$
$\mathrm{X}(1)=\operatorname{SRIMIN}(\mathrm{B}(1), \mathrm{B}(2))$ 


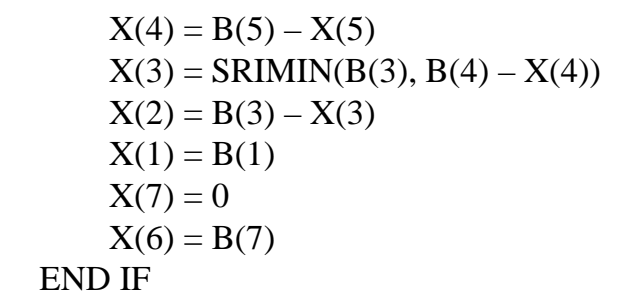

END SUB

SUB PRINTALL

CLS

PRINT " 1"; TAB(7); " 2"; TAB(14); " 3"; TAB(21); " 4"; ТAB(28); " 5"; ТАВ(35); " 6"; ТAB(42); " 7"; TAB(49); " SUM"

PRINT

PRINT

PRINT RI(1); TAB(7); RI(2); TAB(14); RI(3);

TAB(21); RI(4); TAB(28); RI(5); TAB(35); RI(6);

TAB(42); RI(7); TAB(49); SUMRi

PRINT R1(1); TAB(7); R1(2); TAB(14); R1(3);

$\mathrm{TAB}(21) ; \mathrm{R} 1(4) ; \mathrm{TAB}(28) ; \mathrm{R} 1(5) ; \mathrm{TAB}(35) ; \mathrm{R} 1(6) ;$

TAB(42); R1(7); TAB(49); SUMR(1)

PRINT R2(1); TAB(7); R2(2); TAB(14); R2(3);

$\mathrm{TAB}(21) ; \mathrm{R} 2(4) ; \mathrm{TAB}(28) ; \mathrm{R} 2(5) ; \mathrm{TAB}(35) ; \mathrm{R} 2(6) ;$

TAB(42); R2(7); TAB(49); SUMR(2)

PRINT R3(1); TAB(7); R3(2); TAB(14); R3(3);

TAB(21); R3(4); TAB(28); R3(5); $\mathrm{TAB}(35) ; \mathrm{R} 3(6) ;$

TAB(42); R3(7); TAB(49); SUMR(3)

PRINT R4(1); TAB(7); R4(2); TAB(14); R4(3);

$\mathrm{TAB}(21) ; \mathrm{R} 4(4) ; \mathrm{TAB}(28) ; \mathrm{R} 4(5) ; \mathrm{TAB}(35) ; \mathrm{R} 4(6) ;$

TAB(42); R4(7); TAB(49); SUMR(4)

PRINT R5(1); TAB(7); R5(2); TAB(14); R5(3);

TAB(21); R5(4); TAB(28); R5(5); TAB(35); R5(6);

TAB(42); R5(7); TAB(49); SUMR(5)

PRINT R6(1); TAB(7); R6(2); TAB(14); R6(3);

TAB(21); R6(4); TAB(28); R6(5); $\mathrm{TAB}(35) ; \mathrm{R} 6(6) ;$

TAB(42); R6(7); TAB(49); SUMR(6)

PRINT R7(1); TAB(7); R7(2); TAB(14); R7(3);

TAB(21); R7(4); TAB(28); R7(5); TAB(35); R7(6);

TAB(42); R7(7); TAB(49); SUMR(7)

PRINT

PRINT "WORKFORCE = ", WF

PRINT

PRINT "X1 =", X(1), "B1 = ", B(1)

PRINT "X2 =", X(2), "B2 = ", B(2)

PRINT "X3 =", X(3), "B3 = ", B(3)

PRINT "X4 =", X(4), "B4 = ", B(4)

PRINT "X5 =", X(5), "B5 = ", B(5)

PRINT "X6 =", X(6), "B6 = ", B(6)
PRINT "X7 =", X(7), "B7 = ", B(7)

END SUB

FUNCTION RMIN (A, B, C)

IF $\mathrm{A}<=\mathrm{B}$ AND $\mathrm{A}<=\mathrm{C}$ THEN

$\mathrm{RMIN}=\mathrm{A}$

ELSEIF $\mathrm{B}<\mathrm{A}$ AND $\mathrm{B}<=\mathrm{C}$ THEN

$$
\text { RMIN = B }
$$

ELSE

$$
\text { RMIN }=\mathrm{C}
$$

END IF

END FUNCTION

FUNCTION SRIMIN (A, B)

IF A <= B THEN

SRIMIN $=\mathrm{A}$

ELSE

SRIMIN $=\mathrm{B}$

END IF

END FUNCTION

SUB WORKFORCE

IF RiMAX >= SUMRi5 AND RiMAX >= SUMRMAX THEN

$\mathrm{WF}=$ RiMAX

CALL CALBS

CALL XR

CALL PRINTALL

ELSEIF SUMRi5 > RiMAX AND SUMRi5 >= SUMRMAX THEN

$\mathrm{WF}=$ SUMRi5

FLAG = "FALSE"

IF FLAG $=$ "TRUE" THEN

INCR $=5 *$ WF - SUMRi

SUMRi $=$ SUMRi $+(5 *$ WF - SUMRi $)$

$\mathrm{WF}=$ SUMRi $/ 5$ !

FOR $\mathrm{J}=1$ TO 7

IF $($ RI $(J)+$ INCR $)<=$ RiMAX THEN

$\mathrm{RI}(\mathrm{J})=\mathrm{RI}(\mathrm{J})+\mathrm{INCR}$

CALL DAILYREQUIREMENT

END IF EXIT FOR

NEXT J

END IF

CALL CALBS

CALL XRI

CALL PRINTALL

ELSE

WF $=$ SUMRMAX

FLAG = "FALSE"

IF FLAG = "TRUE" THEN

INCR $=3 *$ WF - SUMRMAX

SUMRMAX $=$ SUMRMAX $+(3 *$ WF -

SUMRMAX) 


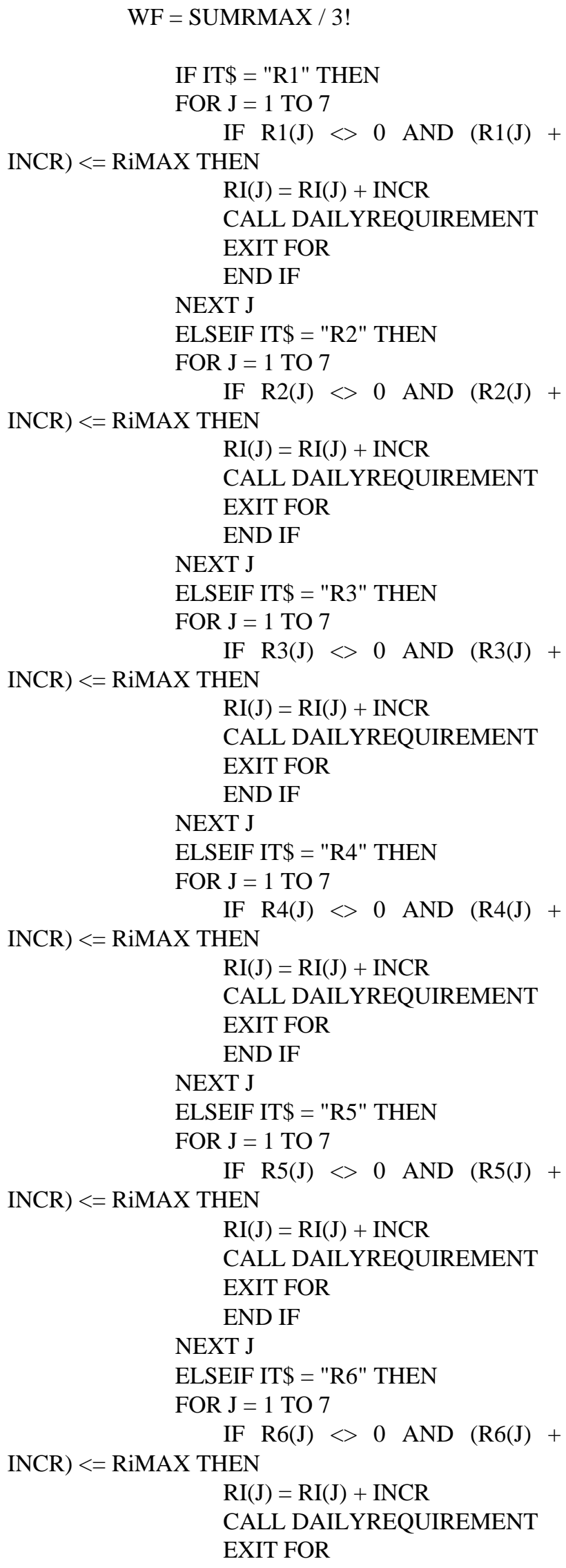

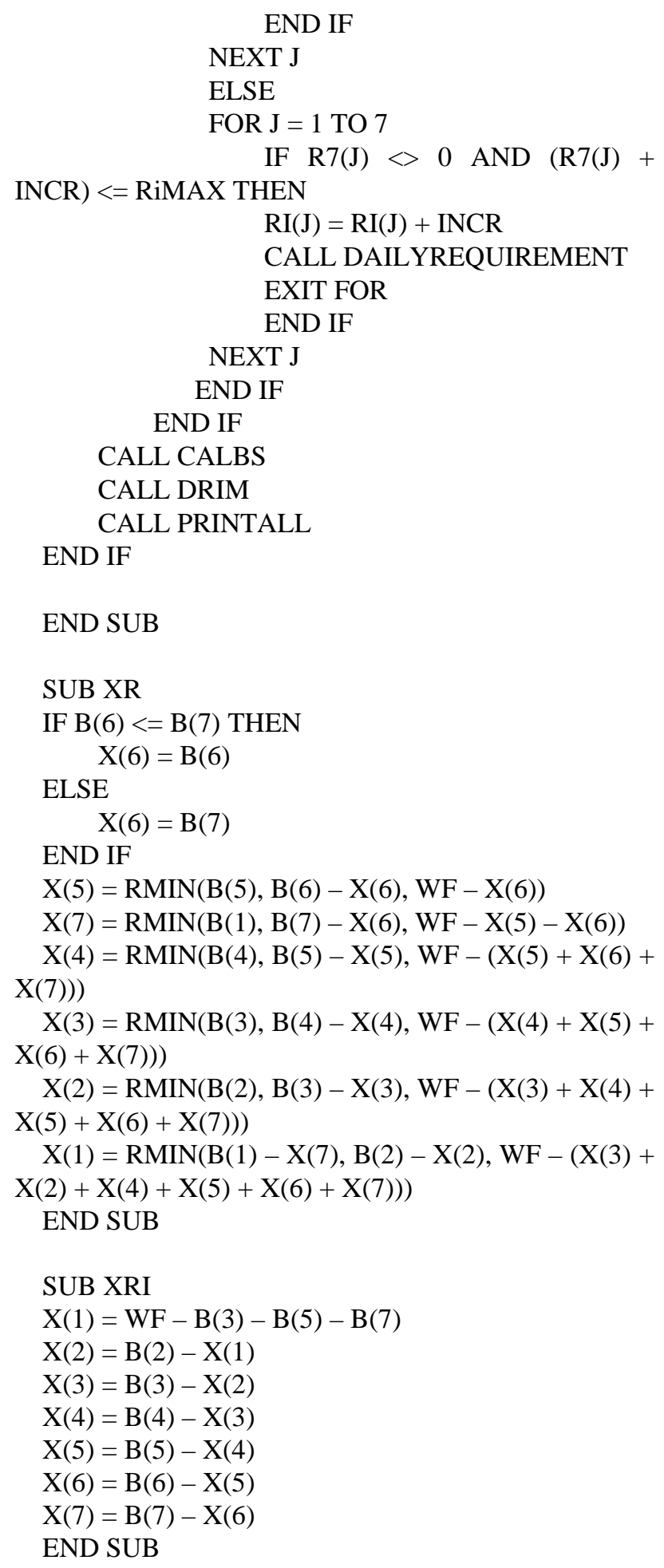

\section{Result and Discussion}

Afam power station Nigeria currently is operating a 5day schedule whereby scheduling workers on weekend (Saturday and Sunday) on overtime basis. To make it possible for her to change to a seven day schedule which is more efficient and less capital intensive, this software 
was developed. The software is still applicable to other organization operating seven days like airline, restaurant and police station.

The workforce size and days-off assignment for Afam power station Nigeria morning shift was determined using this software. The seven morning-time daily labour requirement shown in Table $\mathbf{1}$ were used as input data. The result is shown in Figure 1.

Table 4 below is used to prove if the workforce size of 19 workers can satisfy morning shift daily labour demand in Table 1, Table 4 also represent seven shifts i.e 10 workers are assigned to shift 6 (off on weekends, which is Saturday and Sunday).

However a seven-day schedule (workforce size and days-off assignment) for afternoon or night shifts was generated using the optimization software. The seven afternoon-time or night-time daily labour requirements shown in Table 1 were used as input data. The result is also shown in Figure 2.

Table 5 below is use to prove if the workforce size of 9 workers can satisfy afternoon or night labour demand of Table 1 .

\section{Comparison between Current 5-Day Schedule and 7-Day Schedule [3]}

A 7-day schedule in this paper is a schedule in which each worker must work five days per week, with two

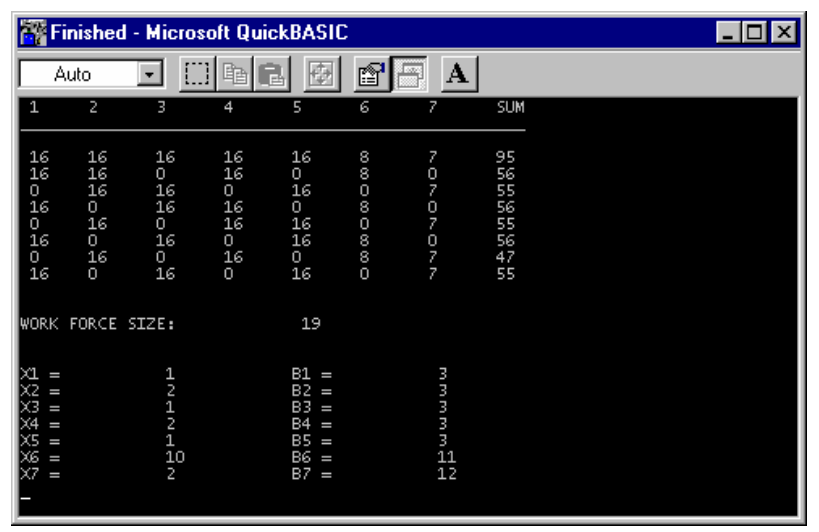

Figure 1. Results of analysis of 7-morning time shift.

Table 4. Total morning daily worker assignment.

\begin{tabular}{ccccccccc}
\hline \multirow{2}{*}{ Day } & \multicolumn{7}{c}{ Morning Shifts } \\
\cline { 2 - 9 } & $\mathrm{x}_{1}$ & $\mathrm{x}_{2}$ & $\mathrm{x}_{3}$ & $\mathrm{x}_{4}$ & $\mathrm{x}_{5}$ & $\mathrm{x}_{6}$ & $\mathrm{x}_{7}$ & Total \\
\hline Monday & $\mathrm{a}$ & 2 & 1 & 2 & 1 & 10 & $\mathrm{a}$ & 16 \\
Tuesday & $\mathrm{a}$ & $\mathrm{a}$ & 1 & 2 & 1 & 10 & 2 & 16 \\
Wednesday & 1 & $\mathrm{a}$ & $\mathrm{a}$ & 2 & 1 & 10 & 2 & 16 \\
Thursday & 1 & 2 & $\mathrm{a}$ & $\mathrm{a}$ & 1 & 10 & 2 & 16 \\
Friday & 1 & 2 & 1 & $\mathrm{a}$ & $\mathrm{a}$ & 10 & 2 & 16 \\
Saturday & 1 & 2 & 1 & 2 & $\mathrm{a}$ & $\mathrm{a}$ & 2 & 8 \\
Sunday & 1 & 2 & 1 & 2 & 1 & $\mathrm{a}$ & $\mathrm{a}$ & 7 \\
\hline
\end{tabular}

Note: $\mathrm{a}=$ off day.

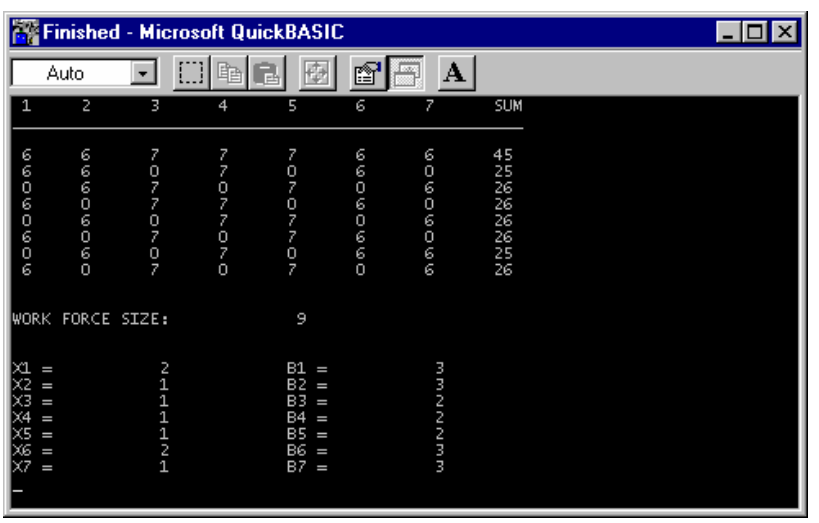

Figure 2. Results of analysis of 7-afternoon time shift.

Table 5. Total afternoon or night daily worker assignment.

\begin{tabular}{ccccccccc}
\hline \multirow{2}{*}{ Day } & \multicolumn{7}{c}{ Afternoon or Night Shifts } \\
\cline { 2 - 9 } & $\mathrm{x}_{1}$ & $\mathrm{x}_{2}$ & $\mathrm{x}_{3}$ & $\mathrm{x}_{4}$ & $\mathrm{x}_{5}$ & $\mathrm{x}_{6}$ & $\mathrm{x}_{7}$ & Total \\
\hline Monday & $\mathrm{a}$ & 1 & 1 & 1 & 1 & 2 & $\mathrm{a}$ & 6 \\
Tuesday & $\mathrm{a}$ & $\mathrm{a}$ & 1 & 1 & 1 & 2 & 1 & 6 \\
Wednesday & 2 & $\mathrm{a}$ & $\mathrm{a}$ & 1 & 1 & 2 & 1 & 7 \\
Thursday & 2 & 1 & $\mathrm{a}$ & $\mathrm{a}$ & 1 & 2 & 1 & 7 \\
Friday & 2 & 1 & 1 & $\mathrm{a}$ & $\mathrm{a}$ & 2 & 1 & 7 \\
Saturday & 2 & 1 & 1 & 1 & $\mathrm{a}$ & $\mathrm{a}$ & 1 & 6 \\
Sunday & 2 & 1 & 1 & 1 & 1 & $\mathrm{a}$ & $\mathrm{a}$ & 6 \\
\hline
\end{tabular}

Note: $\mathrm{a}=$ off day.

consecutive days off. While 5 day schedule workers are schedule on weekend on overtime basis.

\subsection{Current 5-Day Schedule}

The maximum labour demand for the morning shift on weekdays is 16 . Thus, 16 workers are assigned to the morning shift work on the five weekdays (Mo-Fr) on regular-time basis. Similarly, seven workers are assigned to each of the afternoon and night shifts on weekdays on regular time basis. Therefore, the total workforce is equal to $16+7+7=30$ workers. Out of this workforce, $6-8$ employees are assigned to weekend work on overtime basis.

The shift premium pay rate is $110 \%$ for the afternoon shift and $115 \%$ for the night shift. The weekend pay rate for any shift is $50 \%$ higher than the weekdays pay rate. The details of the pay hours calculations are shown in Table 6. The morning shift requires 820 weekly pay hours, while the afternoon and night shift require a total of 942 weekly pay hours. The total workforce size is 30 workers and the weekly pay hours are equal to 1762 .

\subsection{7-Day Workweek Schedule}

This alternative is used to satisfy labour demands with a seven-day workweek schedule for all three work shifts. However, a seven-day schedule is determined for the 
Table 6. Calculation of the total pay hours per week for current 5-day schedule [3].

\begin{tabular}{|c|c|c|c|c|c|}
\hline Work Times & No. of Workers (W) & Hours/day H $=8 \times$ days & Hours/Week T = W $\times$ H & Pay Rate (\%) R & Pay Hours $\mathrm{P}=\mathrm{T} \times \mathrm{R}$ \\
\hline Mo-Fr morning & 16 & 40 & 640 & 100 & 640 \\
\hline Sa morning & 8 & 8 & 64 & 150 & 96 \\
\hline Su morning & 7 & 8 & 56 & 150 & 84 \\
\hline Mo-Fr afternoon & 7 & 40 & 280 & 110 & 308 \\
\hline Sa afternoon & 6 & 8 & 48 & 160 & 76.8 \\
\hline Su afternoon & 6 & 8 & 48 & 160 & 76.8 \\
\hline Mo-Fr night & 7 & 40 & 280 & 115 & 322 \\
\hline Sa night & 6 & 8 & 48 & 165 & 79.2 \\
\hline Su night & 6 & 8 & 48 & 165 & 79.2 \\
\hline
\end{tabular}

morning-shift assignment, using the Quick Basic computer programme for solving $(5,7)$ problem. The seven morning-time daily labour requirements shown in Table 1 were used as input data. The optimum solution requires 19 maintenance workers $(\mathrm{W}=19)$ to satisfy morning labour demands as shown below. Days-off assignments for the 19 morning shift workers are as follows:

$\mathrm{x}_{1}=1, \mathrm{x}_{2}=2, \mathrm{x}_{3}=1, \mathrm{x}_{4}=2, \mathrm{x}_{5}=1, \mathrm{x}_{6}=10, \mathrm{x}_{7}=2$.

Each worker is paid for five workdays per week, at eight hours per day, all at regular time. Hence:

Morning-shift pay hours per week $=19 \times 5 \times 8=760$.

We now consider a seven-day schedule for both the afternoon and night shift assignments. We also use the Quick Basic computer programme for solving $(5,7)$ problem, where the seven afternoon- or night-time daily labour requirements shown in Table $\mathbf{1}$ were used as input data. The optimum solution requires nine workers $(\mathrm{W}=9)$ for each of the afternoon and the night shift, whose days-off assignments are as follows:

$\mathrm{x}_{1}=2, \mathrm{x}_{2}=1, \mathrm{x}_{3}=1, \mathrm{x}_{4}=1, \mathrm{x}_{5}=1, \mathrm{x}_{6}=2, \mathrm{x}_{7}=1$.

In order to calculate the pay hours, it must be noted that the afternoon shift is paid at a premium rate of $110 \%$, while the night shift is paid at a premium rate of $115 \%$.

Afternoon shift pay hours per week $=9 \times 5 \times 8 \times 1.10$ $=396$.

Night shift pay hours per week $=9 \times 5 \times 8 \times 1.15=$ 414.

Adding up the morning-shift assignment, requiring 19 employees and 760 weekly pay hours, we obtain:

Total pay hours per week $=760+396+414=1570$.

Total number of employees $=19+9+9=37$.

Comparison of the two alternative work schedules is shown in Table 7 below. From Table 7 it is obvious that a 7-day schedule, which leads to 11 percent labour cost saving, is the best alternative. Therefore, a 7-schedule was recommended to the management of Afam power station. Though the proposed schedule requires seven more maintenance workers, this is not a serious draw back because Afam power station has an abundant pool of underutilized mechanical maintenance workers. Hence with the current workforce, the proposed schedule can be
Table 7. Comparison of work schedules [3].

\begin{tabular}{ccccc}
\hline $\begin{array}{c}\text { Alternative } \\
\text { work schedule }\end{array}$ & $\begin{array}{c}\text { Pay hours } \\
\text { per week }\end{array}$ & $\begin{array}{c}\text { Savings } \\
\text { in pay hours }\end{array}$ & $\begin{array}{c}\text { Workforce } \\
\text { size }\end{array}$ & $\begin{array}{c}\text { Increase in } \\
\text { workforce }\end{array}$ \\
\hline $\begin{array}{c}\text { Current 5-Day } \\
\text { Schedule }\end{array}$ & 1762 & - & 30 & - \\
$\begin{array}{c}\text { 7-Day Workweek } \\
\text { Schedule }\end{array}$ & 1570 & $10.9 \%$ & 37 & $23.3 \%$ \\
\hline
\end{tabular}

implemented even without employing more maintenance workers.

\section{Conclusions}

The traditional five-day work schedule currently being practiced in the Afam power-station Nigeria is neither efficient nor cost effective. The purpose of this paper is the optimization of the workforce scheduling for solving maintenance problem and improving productivity. In the light of these, this paper has presented a new, efficient optimization software for the cyclic $(5,7)$ days-off scheduling problem.

The software is capable of determining workforce size and assigning workers to days-off pattern for Afam power station and other organization like airline, police station and restaurant operating a 7 day a week. The software does not require specialized training unlike integer programming software.

The software was tested with data from Afam power station Nigeria. Test result shows that the software is capable of generating a seven day schedule. The seven day Schedule is more efficient and cost effective than the five day work schedule. When comparison was made between the existing five-day schedule practiced by Afam power station Nigeria and the seven-day schedule generated by this Software. The seven-day schedule is expected to produce savings of $11 \%$ maintenance labour cost annually [3].

\section{Acknowledgements}

Emovon, Ikuobase would like to thank the managements of the Federal University of Petroleum Resources, Effu- 
run, Nigeria for providing an enabling environment for carrying out this study.

\section{REFERENCES}

[1] H. K. Alfares, “An Efficient Two-Phase Algorithm for Cyclic Days-Off Scheduling,” Computers and Operations Research, Vol. 25, No. 11, 1998, pp. 913-924. doi:10.1016/S0305-0548(98)00033-1

[2] H. K. Alfares, "Efficient Optimization of Cyclic Labour Days-Off Scheduling,” OR Spectrum, Vol. 23, No. 2, 2001, pp. 283-294. doi:10.1007/PL00013353

[3] H. K. Alfares, M. T. Lilly and I. Emovon, "Maintenance Staff Scheduling at Afam Power Station,” Industrial Engineering and Management System, Vol. 6, No. 1, 2007, pp. 22-27.

[4] R. L. Fagbenle, "Prospects and Problems of Solarizing Transport Technology," Nigerian Journal of Renewable Energy, Vol. 2, No. 1, 1991.

[5] H. K. Alfares, "Flexible 4-Day Workweek Scheduling with Weekend Work Frequency Constraints,” Computer and Industrial Engineering, Vol. 44, No. 3, 2003, pp. 325-338. doi:10.1016/S0360-8352(02)00192-4

[6] I. Emovon, "Optimization of Workforce Scheduling for Solving Maintenance Problem in a Power Station (A Case Study of Afam Thermal Power Station),” M.Tech. Thesis, Rivers State University of Science and Technology, Nigeria, 2007. 